\title{
A Meshfree Method for Numerical Solution of Nonhomogeneous Time-Dependent Problems
}

\author{
Ziwu Jiang, ${ }^{1}$ Lingde Su, ${ }^{1,2}$ and Tongsong Jiang ${ }^{1}$ \\ ${ }^{1}$ Department of Mathematics, Linyi University, Linyi 276005, China \\ ${ }^{2}$ College of Mathematics, Shandong Normal University, Jinan 250014, China \\ Correspondence should be addressed to Tongsong Jiang; jiangtongsong@sina.com
}

Received 17 April 2014; Revised 2 July 2014; Accepted 2 July 2014; Published 21 July 2014

Academic Editor: Ali H. Bhrawy

Copyright (c) 2014 Ziwu Jiang et al. This is an open access article distributed under the Creative Commons Attribution License, which permits unrestricted use, distribution, and reproduction in any medium, provided the original work is properly cited.

\begin{abstract}
We propose a new numerical meshfree scheme to solve time-dependent problems with variable coefficient governed by telegraph and wave equations which are more suitable than ordinary diffusion equations in modelling reaction diffusion for such branches of sciences. Finite difference method is adopted to deal with time variable and its derivative, and radial basis functions method is developed for spatial discretization. The results of numerical experiments are presented and are compared with analytical solutions to confirm the accuracy of our scheme.
\end{abstract}

\section{Introduction}

This paper is devoted to the numerical computation of the nonhomogeneous time-dependent problem with the following form:

$$
u_{t t}+a \alpha u_{t}=b f_{1}(x) u_{x x}+c \alpha u+f_{2}(x, t),
$$

where $a, b, c$ are constants, $f_{1}(x)$ and $f_{2}(x, t)$ are given analytic functions, and $\alpha \in\{0,1\}$. The cases $\alpha=0$ and $\alpha=$ 1 correspond to the telegraph problem and wave problem, respectively.

Telegraph equations describe various phenomena in many applied fields, such as a planar random motion of a particle in fluid flow, transmission of electrical impulses in the axons of nerve and muscle cells, propagation of electromagnetic waves in superconducting media, and propagation of pressure waves occurring in pulsatile blood flow in arteries. The wave equation is also an important secondorder linear partial differential equation for the description of waves, such as sound waves, light waves, and water waves. It arises in fields like acoustics, electromagnetics, and fluid dynamics.

Over the past several decades, many numerical methods have been developed to solve boundary-value problems involving ordinary and partial differential equations, such as the finite difference, finite elements, and multigrid methods. As one kind of mesh method, finite difference methods are adopted to solve this partial differential equations $[1,2]$. Although these methods are effective for solving various kinds of partial differential equations, conditional stability of explicit finite difference procedures and the need to use large amount of CPU time in implicit finite difference schemes limit the applicability of these methods. Furthermore, numerical solution can be provided only on mesh points from these methods [3], and the accuracy of these wellknown techniques is reduced in nonsmooth and nonregular domains. Some authors used Legendre-Gauss-Lobatto collocation method and Chebyshev-tau method to solve the spacefractional advection diffusion equation and got high accuracy results $[4,5]$.

Recently, meshfree techniques have attracted attention of researchers in order to avoid the mesh generation. Some meshfree schemes are the element free Galerkin method, the reproducing kernel particle, the point interpolation, and so forth. For more description see [6] and the references therein. Dehghan and Shokri [7] have solved the second order telegraph equations with constant coefficients using meshfree method. In this paper, we extend this problem considered in [7] to one kind of partial differential equations with variable coefficients. 
Radical basis functions (RBFs) method is known as a powerful approximating tool for scattered data interpolation problem. As a meshfree method, the usage of RBFs to solve numerical solution of partial differential equations is based on the collocation scheme. The major advantage of numerical procedures by using RBFs is meshfree compared with the traditional techniques. RBFs are used actively for solving PDEs, and the examples can be found in [10-14].

In the last decade, the development of the RBFs method as a truly meshfree approach for approximating the solutions of partial derivative equations has drawn the attention of many researchers in science and engineering. Meshfree method has become an important numerical computation method, and there are many academic monographs published [15-21].

In this paper, we present an effective numerical scheme to solve time-dependent problems governed by telegraph and wave equations using the meshfree method with RBFs. The results of numerical experiments are presented and are compared with analytical solutions to confirm the good accuracy of the presented scheme.

The layout of the paper is as follows. In Section 2, the overview about RBFs and the numerical scheme of our method on the time-dependent problems are introduced. The results of numerical experiments are presented in Section 3. Section 4 is dedicated to a brief conclusion.

\section{The Meshfree Method}

2.1. Radial Basis Function Approximation. The approximation of a distribution $u(\mathbf{x})$, using RBFs, may be written as a linear combination of $N$ radial basis functions, and usually it takes the following form:

$$
u(\mathbf{x}) \simeq \sum_{j=1}^{N} \lambda_{j} \varphi\left(\mathbf{x}, \mathbf{x}_{j}\right)+\psi(\mathbf{x}), \quad \text { for } \mathbf{x} \in \Omega \subset R^{d},
$$

where $N$ is the number of data points, $\mathbf{x}=\left(x_{1}, x_{2}, \ldots, x_{d}\right)$, $d$ is the dimension of the problem, the $\lambda$ 's are coefficients to be determined, and $\varphi$ is the radial basis function. Equation (2) can be written without the additional polynomial $\psi$. In that case, $\varphi$ must be a positive definite function to guarantee the solvability of the resulting system. However, $\psi$ is usually required when $\varphi$ is conditionally positive definite, that is, when $\varphi$ has a polynomial growth towards infinity. We will use RBFs, which defined as

$$
\begin{array}{r}
\text { Inverse multiquadric (IMQ): } \varphi\left(\mathbf{x}, \mathbf{x}_{j}\right)=\frac{1}{\sqrt{r_{j}^{2}+c^{2}}}, \\
c>0,
\end{array}
$$

Thin plate splines (TPS): $\varphi\left(\mathbf{x}, \mathbf{x}_{j}\right)=r_{j}^{2 m} \log \left(r_{j}\right)$,

$$
m=1,2,3, \ldots,
$$

where $r_{j}=\left\|\mathbf{x}-\mathbf{x}_{j}\right\|$ is the Euclidean norm. Since $\varphi$ given by (3) is $C^{\infty}$ continuous, we can use it directly.

The IMQ radial basis function takes the form $1 / \sqrt{r^{2}+c^{2}}$, $c>0$. The accuracy of the numerical solution is severely influenced by the choice of parameter $c$, since unsuitable parameter $c$ will produce the singular interpolation matrix. Moreover, the number of the chosen nodes can also affect the accuracy. Further learning about RBFs method can be got from $[22,23]$.

If $\mathscr{P}_{q}^{d}$ denotes the space of $d$-variate polynomial of order not exceeding more than $q$ and letting the polynomials $P_{1}, P_{2}, \ldots, P_{m}$ be the basis of $P_{q}^{d}$ in $R^{d}$, then the polynomial $\psi(\mathbf{x})$ in (2) is usually written in the following form:

$$
\psi(\mathbf{x})=\sum_{i=1}^{m} \zeta_{i} P_{i}\left(\mathbf{x}_{j}\right),
$$

where $m=(q-1+d) ! /(d !(q-1) !)$

To get the coefficients $\left(\lambda_{1}, \lambda_{2}, \ldots, \lambda_{N}\right)$ and $\left(\zeta_{1}, \zeta_{2}, \ldots, \zeta_{m}\right)$, the collocation method is used. However, in addition to the $N$ equations resulting from collocating (2) at the $N$ points, extra $m$ equations are required. This is ensured by the $m$ conditions

$$
\sum_{j=1}^{N} \lambda_{j} P_{i}\left(\mathbf{x}_{j}\right)=0, \quad i=1,2, \ldots, m .
$$

Supposed that $\mathscr{L}$, is a linear partial differential operator, then $\mathscr{L} u$ can be approximated by

$$
\mathscr{L} u(\mathbf{x}) \simeq \sum_{j=1}^{N} \lambda_{j} \mathscr{L} \varphi\left(\mathbf{x}, \mathbf{x}_{j}\right)+\mathscr{L} \psi(\mathbf{x}) .
$$

2.2. Nonhomogeneous Time-Dependent Problems. Let ue consider the following time-dependent problem:

$$
\begin{gathered}
u_{t t}+a \alpha u_{t}=b f_{1}(x) u_{x x}+c \alpha u+f_{2}(x, t), \\
x \in \Omega \cup \partial \Omega=\left[a_{1}, b_{1}\right] \subset R, \quad 0<t \leq T,
\end{gathered}
$$

with initial conditions

$$
\begin{array}{ll}
u(x, 0)=g_{1}(x), & x \in \Omega, \\
u_{t}(x, 0)=g_{2}(x), & x \in \Omega,
\end{array}
$$

and Dirichlet boundary conditions

$$
u(x, t)=h(x, t), \quad x \in \partial \Omega, 0<t \leq T,
$$

where $a, b$, and $c$ are constant coefficients, $f_{1}(x), f_{2}(x, t)$, $g_{1}(x), g_{2}(x)$, and $h(x, t)$ are given functions, and $u(x, t)$ is the unknown function.

Equation (7) is discretized according to the following $\theta$ weighted scheme:

$$
\begin{aligned}
& \frac{u(x, t+d t)-2 u(x, t)+u(x, t-d t)}{(d t)^{2}} \\
& \quad+a \alpha \frac{u(x, t+d t)-u(x, t-d t)}{2 d t} \\
& =\theta\left[b f_{1}(x) \Delta u(x, t+d t)+c \alpha u(x, t+d t)\right] \\
& \quad+(1-\theta)\left[b f_{1}(x) \Delta u(x, t)+c \alpha u(x, t)\right]+f_{2}(x, t+d t),
\end{aligned}
$$


where $0 \leq \theta \leq 1, d t$ is the time step size, and $\Delta$ is the Laplace operator. By using the notation $u^{n}=u\left(x, t^{n}\right)$ with $t^{n}=t^{n-1}+$ $d t$, we can get

$$
\begin{aligned}
(1+ & \left.a \alpha \frac{d t}{2}-c \alpha \theta d t^{2}\right) u^{n+1}-b \theta d t^{2} f_{1}(x) \Delta u^{n+1} \\
= & \left(2+c \alpha(1-\theta) d t^{2}\right) u^{n}+b(1-\theta) d t^{2} f_{1}(x) \Delta u^{n} \\
& +\left(a \alpha \frac{d t}{2}-1\right) u^{n-1}+d t^{2} f_{2}^{n+1} .
\end{aligned}
$$

Suppose that there are a total of $N-2$ interpolation points, and $u^{n}(x)$ can be approximated by

$$
u^{n}(x)=\sum_{j=1}^{N-2} \lambda_{j}^{n} \varphi\left(r_{j}\right)+\lambda_{N-1}^{n} x+\lambda_{N}^{n} .
$$

In order to determine the coefficients $\left(\lambda_{1}, \lambda_{2}, \ldots, \lambda_{N-1}, \lambda_{N}\right)$, the collocation method is used by applying (12) at every point $x_{i}, i=1,2, \ldots, N-2$. Thus we obtain

$$
u^{n}\left(x_{i}\right)=\sum_{j=1}^{N-2} \lambda_{j}^{n} \varphi\left(r_{i j}\right)+\lambda_{N-1}^{n} x_{i}+\lambda_{N}^{n}
$$

where $r_{i j}=\sqrt{\left(x_{i}-x_{j}\right)^{2}}$. The additional conditions due to (5) can be written as

$$
\sum_{j=1}^{N-2} \lambda_{j}^{n}=\sum_{j=1}^{N-2} \lambda_{j}^{n} x_{j}=0 .
$$

Writing (13) together with (14) in a matrix form

$$
[u]^{n}=\mathbf{A}[\lambda]^{n},
$$

where

$$
\begin{aligned}
& {[u]^{n}=\left[u_{1}^{n}, u_{2}^{n}, \ldots, u_{N-2}^{n}, 0,0\right]^{T},} \\
& {[\lambda]^{n}=\left[\lambda_{1}^{n}, \lambda_{2}^{n}, \ldots, \lambda_{N}^{n}\right]^{T},}
\end{aligned}
$$

and $\mathbf{A}=\left[a_{i j}, 1 \leq i, j \leq N\right]$ is given as follows:

$$
\mathbf{A}=\left(\begin{array}{ccccc}
\varphi_{11} & \cdots & \varphi_{1(N-2)} & x_{1} & 1 \\
\vdots & \ddots & \vdots & \vdots & \vdots \\
\varphi_{(N-2) 1} & \cdots & \varphi_{(N-2)(N-2)} & x_{N-2} & 1 \\
x_{1} & \cdots & x_{N-2} & 0 & 0 \\
1 & \cdots & 1 & 0 & 0
\end{array}\right)
$$

Assuming that there are $p<N-2$ internal points and $N-2-p$ boundary points, then the $N \times N$ matrix $\mathbf{A}$ can be split into $\mathbf{A}=\mathbf{A}_{d}+\mathbf{A}_{b}+\mathbf{A}_{e}$, where

$$
\begin{aligned}
\mathbf{A}_{d}= & {\left[a_{i j} \text { for }(1 \leq i \leq p, 1 \leq j \leq N), 0 \text { elsewhere }\right], } \\
\mathbf{A}_{b}= & {\left[a_{i j} \text { for }(p+1 \leq i \leq N-2,1 \leq j \leq N),\right.} \\
& 0 \text { elsewhere }], \\
\mathbf{A}_{e}= & {\left[a_{i j} \text { for }(N-1 \leq i \leq N, 1 \leq j \leq N), 0 \text { elsewhere }\right] . }
\end{aligned}
$$

Using the notation $\mathscr{L} \mathbf{A}$ to designate the matrix of the same dimension as $\mathbf{A}$ and containing the elements $\widehat{a}_{i j}$ where $\widehat{a}_{i j}=\mathscr{L} a_{i j}, 1 \leq i, j \leq N$, then (11) together with the boundary conditions (9) can be written in matrix form as

$$
\begin{aligned}
B[\lambda]^{n+1}= & C[\lambda]^{n}+\left(\frac{a \alpha d t}{2}-1\right)\left[u_{d}\right]^{n-1} \\
& +d t^{2}\left[f_{2}\right]^{n+1}+[H]^{n+1},
\end{aligned}
$$

where

$$
\begin{gathered}
C=\left(2+c \alpha(1-\theta) d t^{2}\right) \mathbf{A}_{d}+b(1-\theta) d t^{2}\left(\left[f_{1}\right] * \Delta \mathbf{A}_{d}\right), \\
B=\left(1+a \alpha \frac{d t}{2}-c \alpha \theta d t^{2}\right) \mathbf{A}_{d} \\
-b \theta d t^{2}\left(\left[f_{1}\right] * \Delta \mathbf{A}_{d}\right)+\mathbf{A}_{b}+\mathbf{A}_{e}, \\
{\left[u_{d}\right]^{n-1}=\left[u_{1}^{n-1}, \ldots, u_{p}^{n-1}, 0, \ldots, 0\right]^{T},} \\
{[H]^{n+1}=\left[0, \ldots, 0, h_{p+1}^{n+1}, \ldots, h_{N-2}^{n+1}, 0,0\right]^{T},} \\
{\left[f_{2}\right]^{n+1}=\left[f_{2}^{n+1}\left(x_{1}\right), \ldots, f_{2}^{n+1}\left(x_{p}\right), 0, \ldots, 0\right]^{T},} \\
{\left[f_{1}\right]=\left[f_{1}\left(x_{1}\right), \ldots, f_{1}\left(x_{p}\right), 0, \ldots, 0\right]^{T} .}
\end{gathered}
$$

The operator "*" means that the $i$ th component of vector $\left[f_{1}\right]$ is multiplied to all components of $i$ th row of matrix $\Delta A_{d}$. Equation (19) is obtained by combining (11) applied to the domain points, and (9) applied to the boundary points meanwhile.

At $n=0$, (19) has the following form:

$$
B[\lambda]^{1}=C[\lambda]^{0}+\left(\frac{a \alpha d t}{2}-1\right)\left[u_{d}\right]^{-1}+d t^{2}\left[f_{2}\right]^{1}+[H]^{1} .
$$

To approximate $u^{-1}$, the second initial condition can be used. For this purpose, the second initial condition is discretized as

$$
\frac{u^{1}(x)-u^{-1}(x)}{2 d t}=g_{2}(x), \quad x \in \Omega .
$$

Writing (21) together with (22), we have

$$
\begin{aligned}
(B+ & \left.\left(1-\frac{a \alpha d t}{2}\right) A_{d}\right)[\lambda]^{1} \\
& =C[\lambda]^{0}+(2-a \alpha d t) d t[G]+(d t)^{2}[f]^{1}+[H]^{1},
\end{aligned}
$$

where $[G]=\left[\left(g_{2}\right)_{1}, \ldots,\left(g_{2}\right)_{p}, 0, \ldots, 0\right]^{T}$. Together with the initial condition (8) and (19), we can get all the $\lambda$; thus we can get the numerical solutions.

Since the coefficient matrix is unchanged in each temporal step, we use the $\mathrm{LU}$ factorization to the coefficient matrix only once and use this factorization in our algorithm.

Remark. Although (19) is valid for any value of $\theta \in[0,1]$, we will use $\theta=1 / 2$ (the famous Crank-Nicolson scheme). 

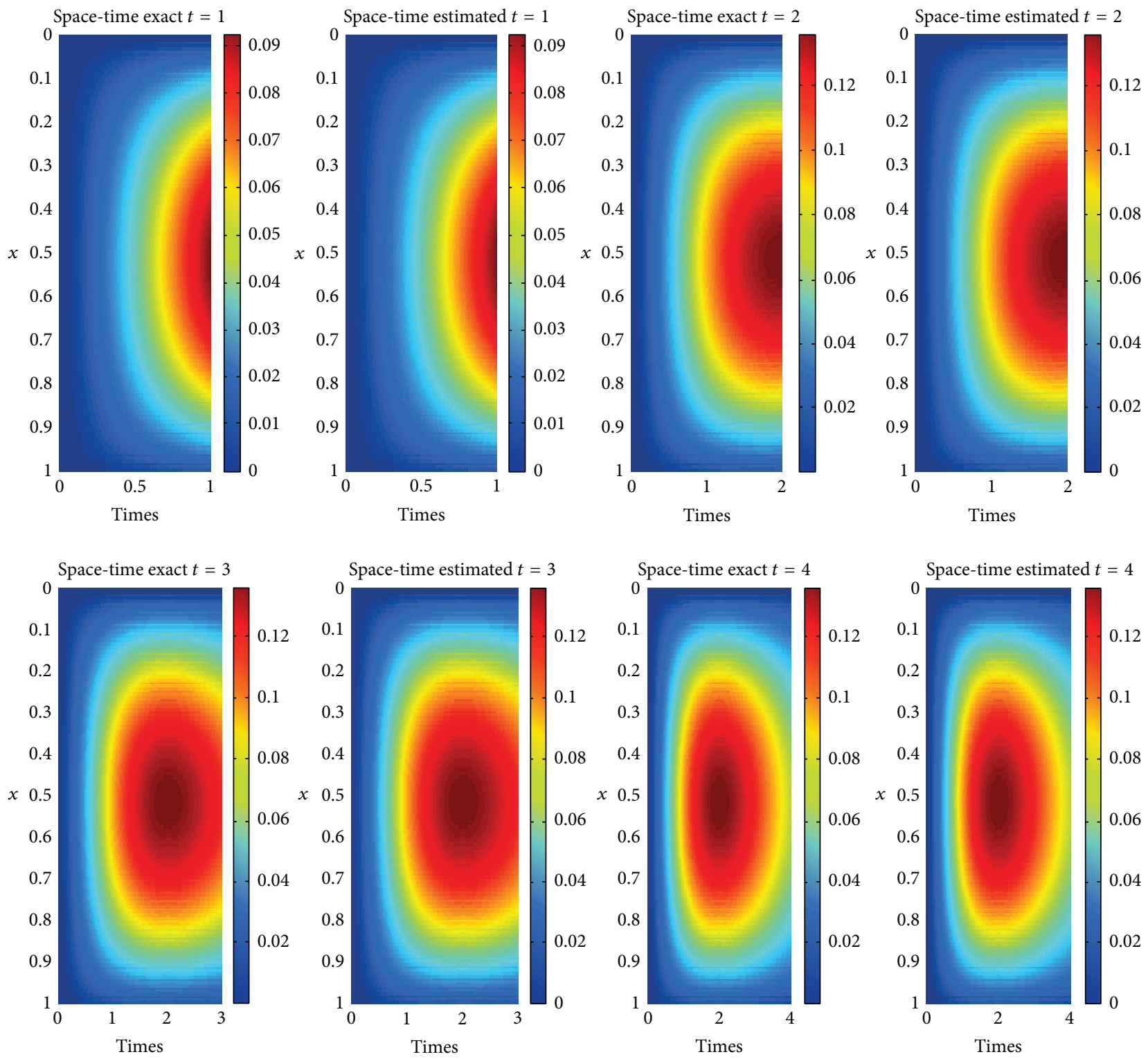

FIGURE 1: Space-time graph of numerical and analytical solutions at time $t=1,2,3,4$ with $d x=0.01, d t=0.001$ and using IMQ (with $c=0.09$ ) as the radial basis function for Example 1.

\section{Numerical Example}

In this section, we present some numerical results to test the efficiency of the new scheme for solving time-dependent problems governed by telegraph and wave equations.

3.1. Example 1. Let $a=b=1, \alpha=1, c=-1$, and $f_{1}(x)=e^{x}$; (1) becomes the telegraph equation

$$
u_{t t}+u_{t}=e^{x} u_{x x}-u+f_{2}(x, t), \quad 0<x<1, t>0
$$

where $f_{2}(x, t)=\left[x(1-x)\left(2-2 t+t^{2}\right)+2 e^{x} t^{2}\right] e^{-t}$, with the boundary conditions

$$
\begin{aligned}
& u(0, t)=0, \quad t>0, \\
& u(1, t)=0, \quad t>0,
\end{aligned}
$$

and the initial conditions

$$
\begin{array}{ll}
u(x, 0)=0, & 0 \leq x \leq 1, \\
u_{t}(x, 0)=0, & 0 \leq x \leq 1 .
\end{array}
$$

The analytical solution of the equation is

$$
u(x, t)=x(1-x) t^{2} e^{-t} .
$$



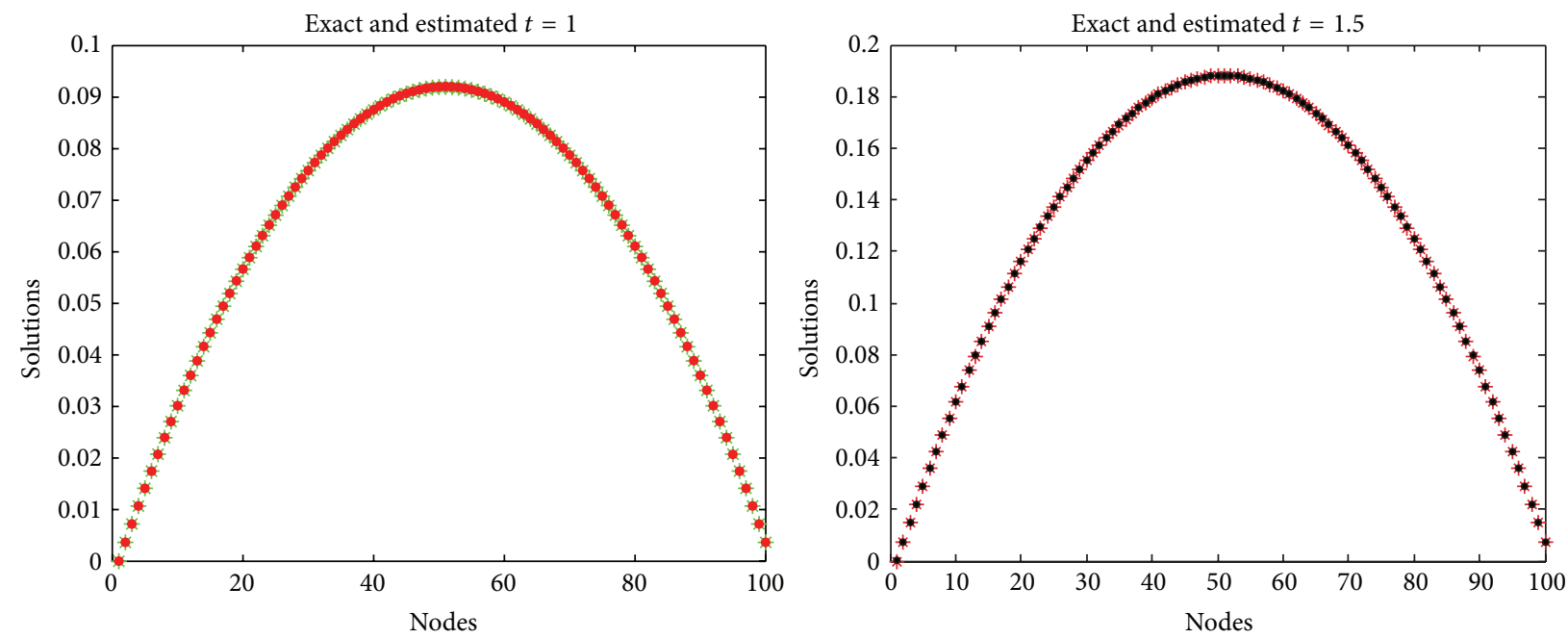

* Exact

- Estimated

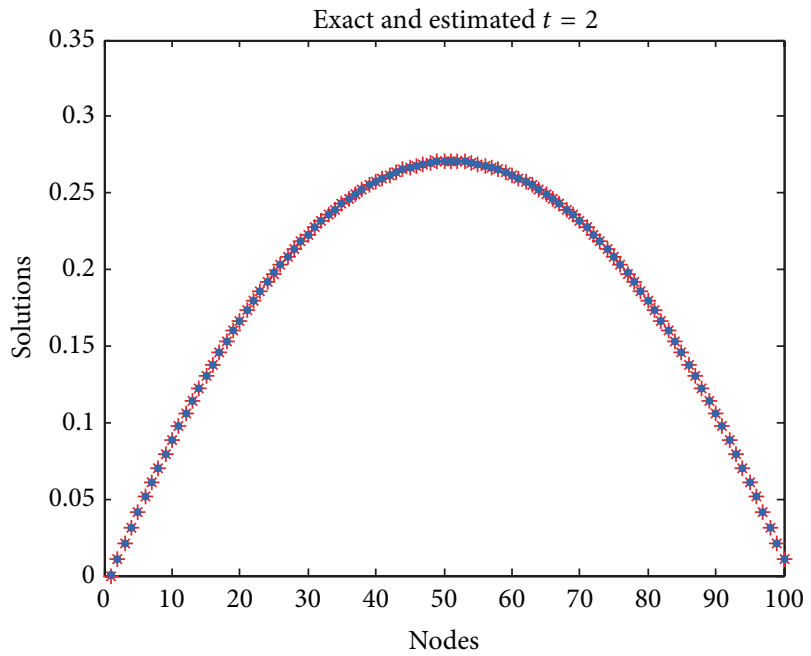

* Exact

- Estimated

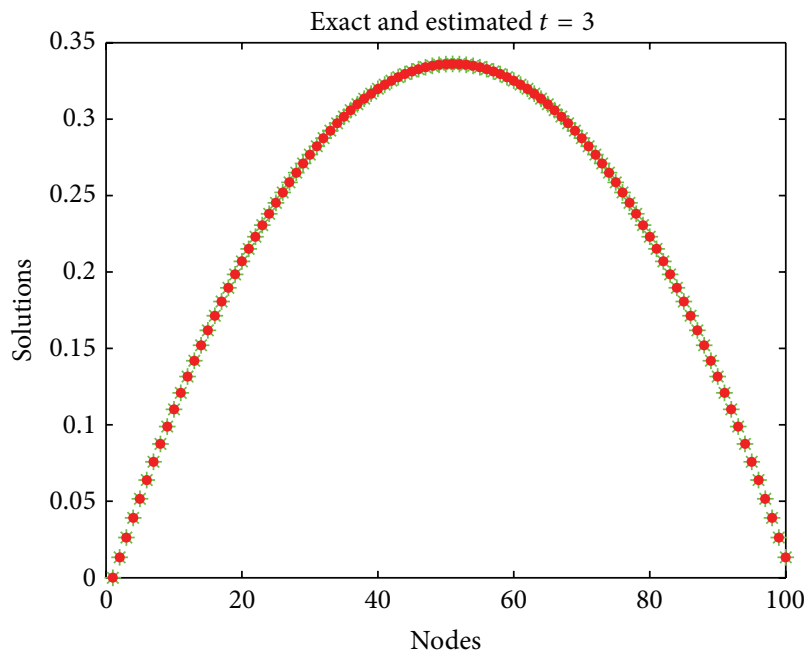

* Exact

- Estimated

* Exact

- Estimated

FIGURE 2: The numerical and analytical solutions at time $t=1,1.5,2,3$ with $d x=0.01, d t=0.001$ and using TPS (with $m=2$ ) as the radial basis function for Example 2.

We solve this problem by using the IMQ and TPS radial basis functions. These results are obtained for $d x=0.01$ and $d t=0.001$. The $L_{\infty}, L_{2}$, and root-mean-square (RMS) errors are obtained in Table 1 for $t=1,2,3$, and 4 .

We also give the analysis of the parameter $c$ in IMQ for the results. In Table 2 , the $L_{\infty}, L_{2}$, and RMS errors with different $c$ at time $t=3$ are presented.

The space-time graph of analytical and numerical results for $t=1,2,3,4$ with $d x=0.01, d t=0.001, \theta=1 / 2$ by using IMQ (with $c=0.09$ ) as the RBF is given in Figure 1 . The results obtained show the very good accuracy and efficiency of the new approximate scheme. Note that we cannot distinguish the exact solution from the estimated solution in Figure 1.
3.2. Example 2. In this example, $\alpha=0, b=1$, and $f_{1}(x)=$ $x^{2}+1$ (1) become the wave equation

$$
u_{t t}=\left(x^{2}+1\right) u_{x x}+f_{2}(x, t), \quad 0<x<1, t>0,
$$

where $f_{2}(x, t)=\left[2\left(x^{2}+1\right) t^{3}+\left(x-x^{2}\right)\left(6 t-6 t^{2}+t^{3}\right)\right] e^{-t}$, with the boundary conditions

$$
\begin{aligned}
& u(0, t)=0, \quad t>0, \\
& u(1, t)=0, \quad t>0 .
\end{aligned}
$$



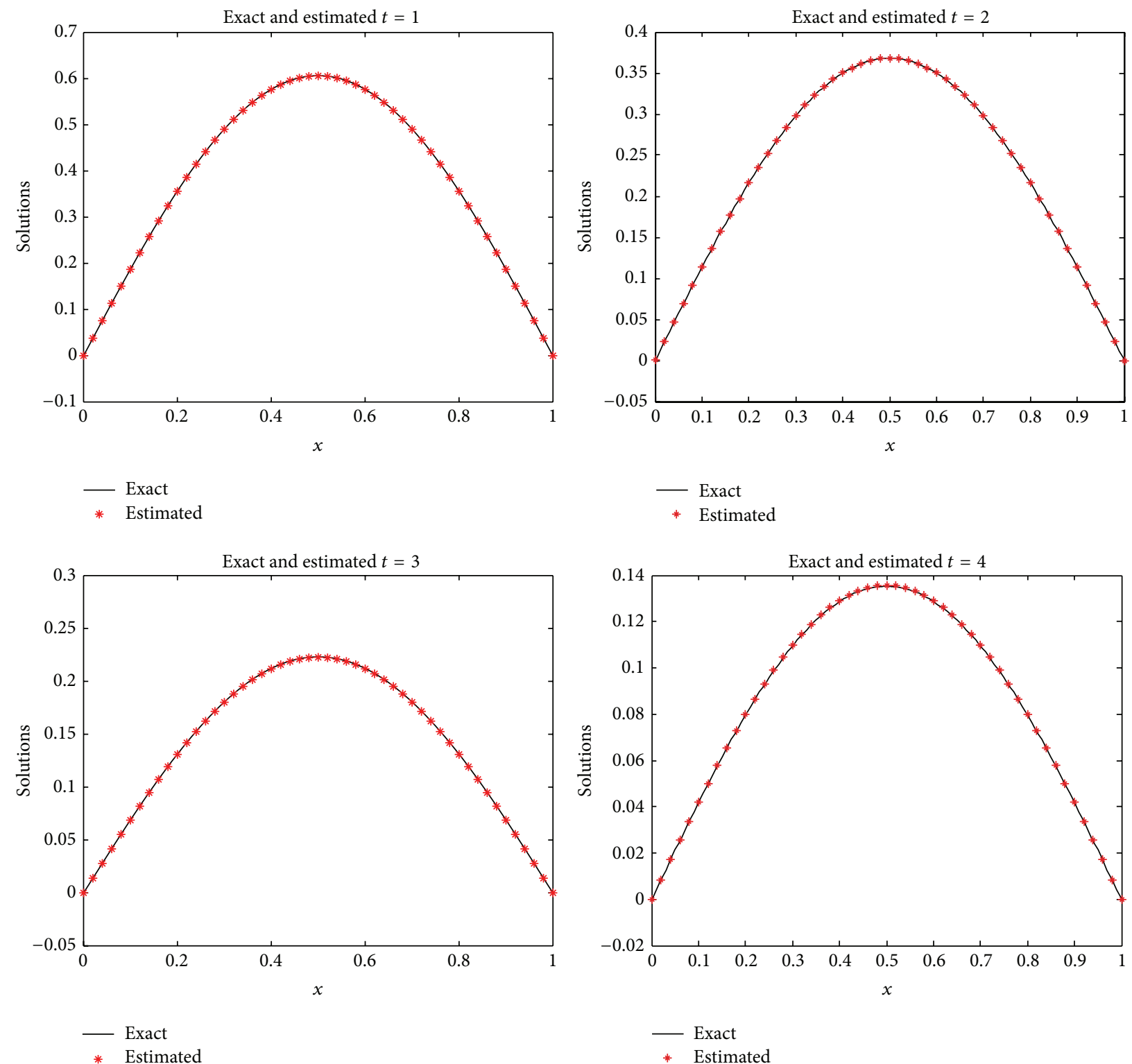

FIGURE 3: Space-time graph of numerical and analytical solutions at time $t=1,2,3,4$ with $d x=0.01, d t=0.001$ and using IMQ (with $c=0.08)$ as the radial basis function for Example 3 .

The initial conditions are given by

$$
\begin{array}{cc}
u(x, 0)=g_{1}(x)=0, & 0 \leq x \leq 1, \\
u_{t}(x, 0)=g_{2}(x)=0, & 0 \leq x \leq 1,
\end{array}
$$

and the analytical solution of the equation is given as

$$
u(x, t)=\left(x-x^{2}\right) t^{3} e^{-t} .
$$

IMQ and TPS are used as the radial basis function in the discussed scheme, and these results are obtained for $d x=$ 0.01 and $d t=0.001$.

Table 3 presents the $L_{\infty}$ and $L_{2}$ and RMS errors for $t=$ $1,1.5,2$, and 3 .

Similar to Example 2, in Table 4, the $L_{\infty}, L_{2}$, and RMS errors with different $c$ at time $t=2$ are presented.
The analytical and numerical results for $t=1,1.5,2$, and 3 with $d x=0.01, d t=0.001, \theta=1 / 2$ are given in Figure 2 by using TPS (with $m=2$ ) as the RBF.

3.3. Example 3. In this example, we consider the following wave equation:

$$
u_{t t}=u_{x x}+f_{2}(x, t), \quad 0<x<1, t>0
$$

where $f_{2}(x, t)=\left((1 / 4)+\pi^{2}\right) e^{-t / 2} \sin (\pi x)$, with the boundary conditions

$$
\begin{aligned}
& u(0, t)=0, \quad t>0, \\
& u(1, t)=0, \quad t>0,
\end{aligned}
$$


TABLE 1: Numerical errors using IMQ $(c=0.09)$ and TPS $(m=2)$ represented at different times, where $d x=0.01, d t=0.001$, and $\theta=1 / 2$.

\begin{tabular}{ccccccc}
\hline & \multicolumn{2}{c}{$L_{\infty}$-error } & \multicolumn{2}{c}{$L_{2}$-error } & \multicolumn{2}{c}{ RMS } \\
& IMQ & TPS & IMQ & TPS & IMQ & $4.778 \times 10^{-5}$ \\
\hline 1 & $6.103 \times 10^{-5}$ & $6.576 \times 10^{-5}$ & $4.401 \times 10^{-5}$ & $4.849 \times 10^{-5}$ & $4.336 \times 10^{-5}$ & $1.580 \times 10^{-5}$ \\
2 & $3.130 \times 10^{-5}$ & $2.403 \times 10^{-5}$ & $2.267 \times 10^{-5}$ & $1.604 \times 10^{-5}$ & $2.233 \times 10^{-5}$ & $2.163 \times 10^{-6}$ \\
3 & $9.933 \times 10^{-6}$ & $4.192 \times 10^{-6}$ & $8.155 \times 10^{-6}$ & $2.195 \times 10^{-6}$ & $8.035 \times 10^{-6}$ & $9.665 \times 10^{-6}$ \\
4 & $1.792 \times 10^{-5}$ & $1.393 \times 10^{-5}$ & $1.351 \times 10^{-5}$ & $9.809 \times 10^{-6}$ & $1.331 \times 10^{-5}$ & \\
\hline
\end{tabular}

TABLE 2: Numerical errors using IMQ represented with different parameter $c$ at time $t=3$, where $d x=0.01, d t=0.001$, and $\theta=1 / 2$.

\begin{tabular}{lccc}
\hline$c$ & $L_{\infty}$ & $L_{2}$ & RMS \\
\hline 0.01 & $9.242 \times 10^{-2}$ & $6.522 \times 10^{-2}$ & $6.427 \times 10^{-2}$ \\
0.03 & $5.959 \times 10^{-4}$ & $5.559 \times 10^{-4}$ & $5.478 \times 10^{-4}$ \\
0.05 & $1.175 \times 10^{-4}$ & $1.139 \times 10^{-4}$ & $1.122 \times 10^{-4}$ \\
0.07 & $2.850 \times 10^{-5}$ & $2.630 \times 10^{-5}$ & $2.591 \times 10^{-5}$ \\
0.09 & $9.933 \times 10^{-6}$ & $8.155 \times 10^{-6}$ & $8.035 \times 10^{-6}$ \\
\hline
\end{tabular}

TABLE 3: Numerical errors using IMQ $(c=0.08)$ and TPS $(m=2)$ represented at different times, where $d x=0.01, d t=0.001$, and $\theta=1 / 2$.

\begin{tabular}{lcccccc}
\hline$t$ & \multicolumn{2}{c}{$L_{\infty}$-error } & \multicolumn{2}{c}{$L_{2}$-error } & \multicolumn{2}{c}{ RMS } \\
& IMQ & TPS & IMQ & TPS & IMQ \\
\hline 1.0 & $7.456 \times 10^{-5}$ & $7.997 \times 10^{-5}$ & $5.319 \times 10^{-5}$ & $5.896 \times 10^{-5}$ & $5.292 \times 10^{-5}$ & $5.867 \times 10^{-5}$ \\
1.5 & $1.711 \times 10^{-5}$ & $3.650 \times 10^{-5}$ & $1.019 \times 10^{-5}$ & $2.839 \times 10^{-5}$ & $1.014 \times 10^{-5}$ & $2.825 \times 10^{-5}$ \\
2.0 & $5.011 \times 10^{-5}$ & $8.559 \times 10^{-5}$ & $3.346 \times 10^{-5}$ & $6.314 \times 10^{-5}$ & $3.329 \times 10^{-5}$ & $6.283 \times 10^{-5}$ \\
3.0 & $5.832 \times 10^{-5}$ & $2.328 \times 10^{-5}$ & $4.935 \times 10^{-5}$ & $1.562 \times 10^{-5}$ & $4.910 \times 10^{-5}$ & $1.554 \times 10^{-5}$ \\
\hline
\end{tabular}

TABLE 4: Numerical errors using IMQ represented with different parameter $c$ at time $t=2$, where $d x=0.01, d t=0.001$, and $\theta=1 / 2$.

\begin{tabular}{lccr}
\hline$c$ & $L_{\infty}$ & $L_{2}$ & RMS \\
\hline 0.01 & $2.260 \times 10^{-1}$ & $1.598 \times 10^{-1}$ & $1.590 \times 10^{-1}$ \\
0.02 & $7.283 \times 10^{-3}$ & $5.736 \times 10^{-3}$ & $5.707 \times 10^{-3}$ \\
0.04 & $6.325 \times 10^{-4}$ & $5.998 \times 10^{-4}$ & $5.969 \times 10^{-4}$ \\
0.06 & $1.261 \times 10^{-4}$ & $8.307 \times 10^{-5}$ & $8.265 \times 10^{-5}$ \\
0.08 & $5.011 \times 10^{-5}$ & $3.346 \times 10^{-5}$ & $3.329 \times 10^{-5}$ \\
\hline
\end{tabular}

TABLE 5: Numerical errors using IMQ $(c=0.08)$ and TPS $(m=2)$ represented at different times, where $d x=0.01, d t=0.001$, and $\theta=1 / 2$.

\begin{tabular}{ccccccc}
\hline$t$ & \multicolumn{2}{c}{$L_{\infty}$-error } & \multicolumn{2}{c}{$L_{2}$-error } & \multicolumn{2}{c}{ RMS } \\
& IMQ & TPS & IMQ & TPS & IMQ \\
\hline 1 & $5.304 \times 10^{-4}$ & $3.784 \times 10^{-4}$ & $4.016 \times 10^{-4}$ & $2.614 \times 10^{-4}$ & $3.996 \times 10^{-4}$ & $2.601 \times 10^{-4}$ \\
2 & $2.078 \times 10^{-4}$ & $1.479 \times 10^{-4}$ & $1.567 \times 10^{-4}$ & $1.022 \times 10^{-4}$ & $1.559 \times 10^{-4}$ & $1.017 \times 10^{-4}$ \\
3 & $4.024 \times 10^{-4}$ & $2.867 \times 10^{-4}$ & $3.039 \times 10^{-4}$ & $1.980 \times 10^{-4}$ & $3.624 \times 10^{-4}$ & $1.917 \times 10^{-4}$ \\
4 & $2.883 \times 10^{-4}$ & $2.015 \times 10^{-4}$ & $2.134 \times 10^{-4}$ & $1.392 \times 10^{-4}$ & $2.124 \times 10^{-4}$ & $1.385 \times 10^{-4}$ \\
5 & $3.545 \times 10^{-4}$ & $2.522 \times 10^{-4}$ & $2.671 \times 10^{-4}$ & $1.742 \times 10^{-4}$ & $2.658 \times 10^{-4}$ & $1.734 \times 10^{-4}$ \\
\hline
\end{tabular}

TABLE 6: Numerical errors using IMQ represented with different parameter $c$ at time $t=1$, where $d x=0.01, d t=0.001$, and $\theta=1 / 2$.

\begin{tabular}{lccc}
\hline$c$ & $L_{\infty}$ & $L_{2}$ & RMS \\
\hline 0.01 & $1.584 \times 10^{-1}$ & $1.056 \times 10^{-1}$ & $1.051 \times 10^{-1}$ \\
0.02 & $3.556 \times 10^{-2}$ & $2.789 \times 10^{-2}$ & $2.775 \times 10^{-2}$ \\
0.04 & $3.344 \times 10^{-3}$ & $3.173 \times 10^{-3}$ & $3.158 \times 10^{-3}$ \\
0.06 & $9.963 \times 10^{-4}$ & $8.535 \times 10^{-4}$ & $8.493 \times 10^{-4}$ \\
0.08 & $5.304 \times 10^{-4}$ & $4.016 \times 10^{-4}$ & $3.996 \times 10^{-4}$ \\
\hline
\end{tabular}



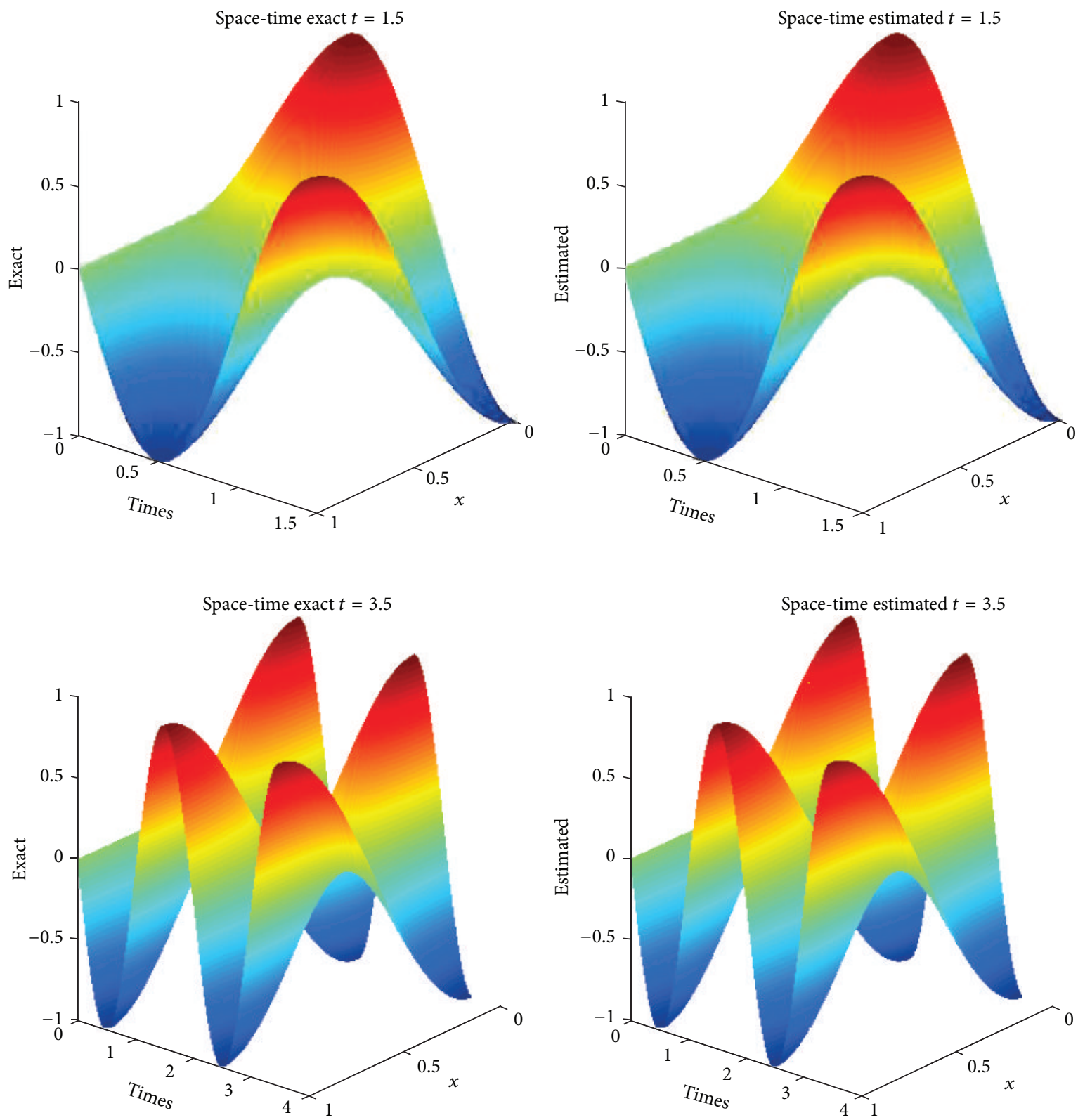

FIGURE 4: Space-time graph of numerical and analytical solutions at time $t=1.5,3.5$ with $d x=0.01, d t=0.001$ and using TPS ( $m=2)$ as the radial basis function for Example 4.

and the initial conditions

$$
\begin{gathered}
u(x, 0)=\sin (\pi x), \quad 0 \leq x \leq 1, \\
u_{t}(x, 0)=-\frac{1}{2} \sin (\pi x), \quad 0 \leq x \leq 1 .
\end{gathered}
$$

The analytical solution of the equation is

$$
u(x, t)=e^{-t / 2} \sin (\pi x)
$$

We also use IMQ and TPS as the radial basis functions in the discussed scheme, and these results are obtained for $d x=0.01$ and $d t=0.001$. The $L_{\infty}, L_{2}$, and RMS errors are obtained in Table 5 for $t=1,2,3,4$ and 5 .
We also give the analysis of the parameter $c$ in IMQ for the results. In Table 6 , the $L_{\infty}, L_{2}$, and RMS errors with different $c$ at time $t=1$ are presented.

The graph of analytical and numerical results for $t=$ $1,2,3$, and 4 with $d x=0.01, d t=0.001$, and $\theta=1 / 2$ and using IMQ (with $c=0.08$ ) as the RBF are given in Figure 3 .

3.4. Example 4. In this example, $b=1, \alpha=0$, and $f_{1}(x)=1$; then (1) becomes the telegraph equation

$$
u_{t t}=u_{x x}+f_{2}(x, t), \quad 0<x<1, t>0
$$



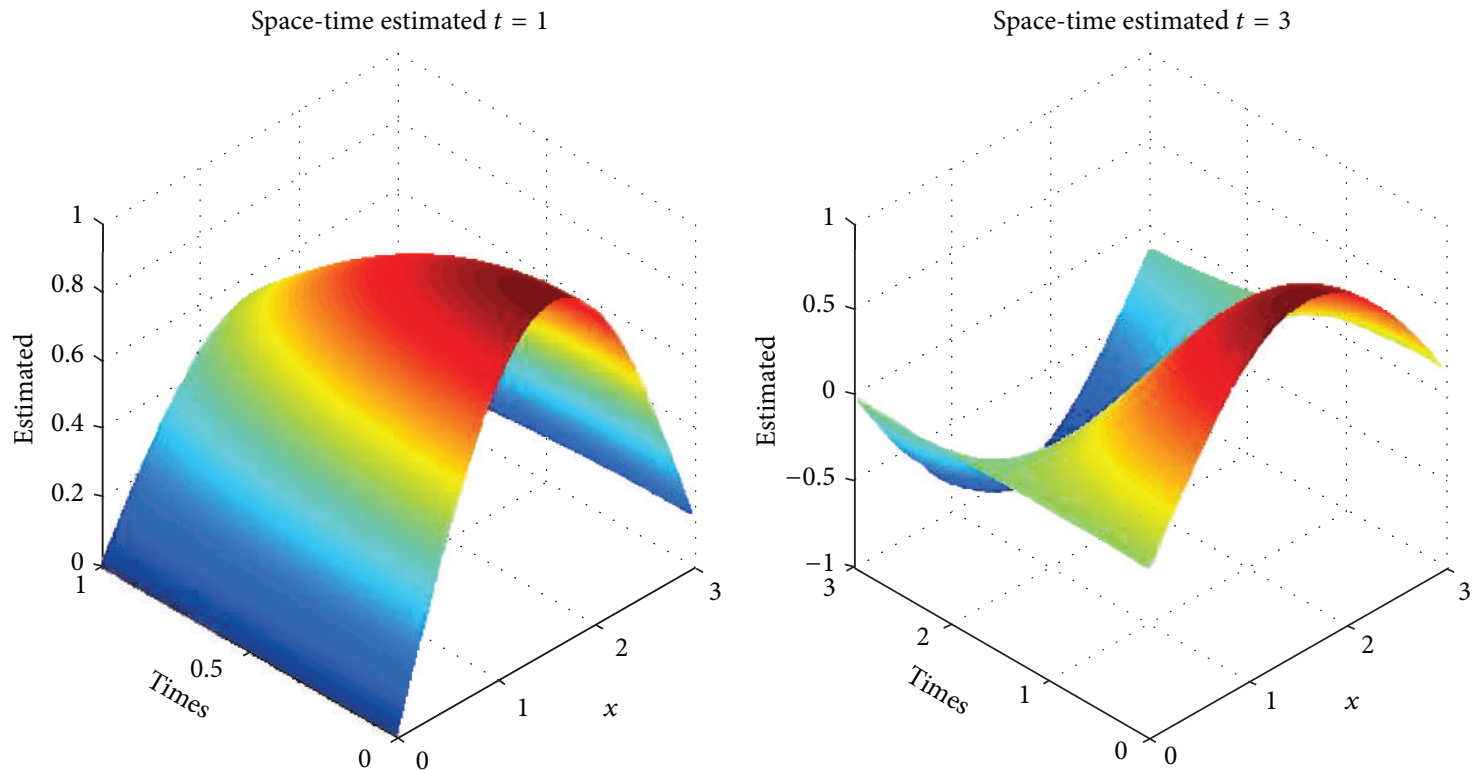

FIGURE 5: Space-time graph of numerical solutions at time $t=1,3$ with $d x=0.01, d t=0.001$ by using IMQ (with $c=0.09$ ) in Example 5 .

TABLE 7: Numerical errors using IMQ $(c=0.06)$ and TPS $(m=2)$ represented at different times, where $d x=0.01, d t=0.001$, and $\theta=1 / 2$.

\begin{tabular}{lcccccc}
\hline \multirow{2}{*}{$t$} & \multicolumn{2}{c}{$L_{\infty}$-error } & \multicolumn{2}{c}{$L_{2}$-error } & \multicolumn{2}{c}{ RMS } \\
& IMQ & TPS & IMQ & TPS & IMQ & $3.185 \times 10^{-4}$ \\
\hline 0.5 & $5.588 \times 10^{-4}$ & $4.458 \times 10^{-4}$ & $4.219 \times 10^{-4}$ & $3.201 \times 10^{-4}$ & $4.198 \times 10^{-4}$ & $3.029 \times 10^{-4}$ \\
1.5 & $3.248 \times 10^{-4}$ & $4.274 \times 10^{-4}$ & $2.189 \times 10^{-4}$ & $3.044 \times 10^{-4}$ & $2.178 \times 10^{-4}$ & $3.122 \times 10^{-4}$ \\
2.5 & $5.514 \times 10^{-4}$ & $4.378 \times 10^{-4}$ & $4.159 \times 10^{-4}$ & $3.138 \times 10^{-4}$ & $4.139 \times 10^{-4}$ & $2.118 \times 10^{-4}$ \\
3.5 & $3.167 \times 10^{-4}$ & $4.195 \times 10^{-4}$ & $2.129 \times 10^{-4}$ & $2.982 \times 10^{-4}$ & $2.967 \times 10^{-4}$ \\
\hline
\end{tabular}

where $f_{2}(x, t)=0$, with the initial conditions

$$
\begin{gathered}
u(x, 0)=0, \quad 0 \leq x \leq 1, \\
u_{t}(x, 0)=\pi \cos (\pi x), \quad 0 \leq x \leq 1 .
\end{gathered}
$$

The analytical solution of the equation is

$$
u(x, t)=\cos (\pi x) \sin (\pi t)
$$

We extracted the boundary conditions from the exact solution.

The IMQ and TPS radial basis functions utilized in our scheme and these results are obtained for $d x=0.01$, and $d t=$ 0.001 . The $L_{\mathrm{\infty}}, L_{2}$, and RMS errors are obtained in Table 7 for $t=0.5,1.5,2.5$ and 3.5.

The space-time graph of analytical and numerical results for $t=1.5,3.5$ with $d x=0.01, d t=0.001$, and $\theta=1 / 2$ and using TPS (with $m=2$ ) as the RBF are given in Figure 4 .

3.5. Example 5. In this example, we consider the following telegraph equation:

$$
u_{t t}+12 u_{t}=u_{x x}-4 u+f_{2}(x, t), \quad 0<x<3, t>0,
$$

where $f_{2}(x, t)=-12 \sin x \sin t+4 \cos t \sin x$, with the boundary conditions

$$
\begin{gathered}
u(0, t)=0, \quad t>0, \\
u(1, t)=\sin 1 \cos t, \quad t>0,
\end{gathered}
$$

and the initial conditions

$$
\begin{gathered}
u(x, 0)=\sin x, \quad 0 \leq x \leq 1, \\
u_{t}(x, 0)=-\sin x \sin t, \quad 0 \leq x \leq 1 .
\end{gathered}
$$

We use the radial basis functions (IMQ, TPS) for the discussed scheme. These results are obtained for $d x=0.01$ and $d t=0.001$.

The space-time graph of numerical results for $t=1,3$ with $d x=0.01, d t=0.001$, and $\theta=1 / 2$ and using IMQ (with $c=0.09$ ) as the RBF are given in Figure 5.

We also compared our methods with quartic B-spline collocation method [8] and cubic B-spline quasi-interpolation method [9], and Tables 8 and 9 give the comparison, respectively.

\section{Conclusions}

In this paper, we proposed a numerical scheme to solve the nonhomogeneous time-dependent problems using the 
TABLE 8: The $L_{\infty}$ errors compared with quartic B-spline collection method at different times with $d x=0.005, d t=0.001$ in $[0,1]$ and IMQ $\operatorname{RBF}(c=0.05)$.

\begin{tabular}{lcccc}
\hline Methods & $t=0.2$ & $t=0.4$ & $t=0.6$ & $t=0.8$ \\
\hline Present Method & $8.853 \times 10^{-5}$ & $1.896 \times 10^{-4}$ & $2.594 \times 10^{-4}$ & $3.028 \times 10^{-4}$ \\
Quartic B-spline [8] & $2.428 \times 10^{-5}$ & $7.932 \times 10^{-5}$ & $1.209 \times 10^{-4}$ & $1.488 \times 10^{-4}$ \\
\hline
\end{tabular}

TABLE 9: The $L_{\infty}$ errors compared with cubic B-spline quasi-interpolation method at different times with $d x=0.002, d t=0.0005$ in $[0,1]$ and IMQ RBF $(c=0.02)$.

\begin{tabular}{lcccc}
\hline Methods & $t=0.4$ & $t=0.6$ & $t=0.8$ & $t=1$ \\
\hline Present Method & $9.505 \times 10^{-5}$ & $1.299 \times 10^{-4}$ & $1.515 \times 10^{-4}$ & $1.616 \times 10^{-4}$ \\
Cubic B-spline [9] & $5.576 \times 10^{-5}$ & $6.933 \times 10^{-4}$ & $7.686 \times 10^{-5}$ & $7.891 \times 10^{-5}$ \\
\hline
\end{tabular}

meshfree method with inverse multiquadric and generalized thin plate splines radial basis functions. The main advantage of this method over finite difference techniques is that the latter methods provide the solution of the problem on mesh points only. Numerical examples are given to demonstrate the validity and applicability of our method. The results show that our method is meshless and accurate. Indeed by selecting an optimal parameter $c$ in the inverse multiquadric function, excellent numerical results are obtained.

\section{Conflict of Interests}

The authors declare that there is no conflict of interests regarding the publication of this paper.

\section{Acknowledgments}

The respected anonymous referees have carefully reviewed this paper. As a result of their careful analysis, our paper has been improved. The authors would like to express their thankfulness to them for their helpful constructive comments. This work was supported by the National Natural Science Foundation of China (Grant nos. 11301252, 11301529, and 11201212) and Applied Mathematics Enhancement Program of Linyi University.

\section{References}

[1] M. Dehghan, "Parameter determination in a partial differential equation from the overspecified data," Mathematical and Computer Modelling, vol. 41, no. 2-3, pp. 196-213, 2005.

[2] M. Dehghan, "Implicit collocation technique for heat equation with non-classic initial condition," International Journal of Nonlinear Sciences and Numerical Simulation, vol. 7, no. 4, pp. 447-450, 2006.

[3] M. Dehghan, "Finite difference procedures for solving a problem arising in modeling and design of certain optoelectronic devices," Mathematics and Computers in Simulation, vol. 71, no. 1, pp. 16-30, 2006.

[4] A. H. Bhrawy and D. Baleanu, "A spectral Legendre-GaussLobatto collocation method for a space-fractional advection diffusion equations with variable coefficients," Reports on Mathematical Physics, vol. 72, no. 2, pp. 219-233, 2013.
[5] R. F. Ren, H. B. Li, W. Jiang, and M. Y. Song, "An efficient Chebyshev-tau method for solving the space fractional diffusion equations," Applied Mathematics and Computation, vol. 224, pp. 259-267, 2013.

[6] G. R. Liu and Y. T. Gu, "Boundary meshfree methods based on the boundary point interpolation methods," Engineering Analysis with Boundary Elements, vol. 28, no. 5, pp. 475-487, 2004.

[7] M. Dehghan and A. Shokri, "A numerical method for solving the hyperbolic telegraph equation," Numerical Methods for Partial Differential Equations, vol. 24, no. 4, pp. 1080-1093, 2008.

[8] M. Dosti and A. Nazemi, "Quartic B-spline collocation method for solving one-dimensional hyperbolic telegraph equation," Journal of Information and Computing Science, vol. 7, no. 2, pp. 83-90, 2012.

[9] M. Dosti and A. Nazemi, "Solving one-dimensional hyperbolic telegraph equation using cubic B-spline quasi-interpolation," World Academy of Science, Engineering and Technology, vol. 5, pp. 930-935, 2011.

[10] E. J. Kansa, "Multiquadrics-a scattered data approximation scheme with applications to computational fluid-dynamics. I. Surface approximations and partial derivative estimates," Computers \& Mathematics with Applications, vol. 19, no. 8-9, pp. $127-145,1990$.

[11] M. Zerroukat, H. Power, and C. S. Chen, "A numerical method for heat transfer problems using collocation and radial basis functions," International Journal for Numerical Methods in Engineering, vol. 42, no. 7, pp. 1263-1278, 1998.

[12] M. Li, T. Jiang, and Y. C. Hon, "A meshless method based on RBFs method for nonhomogeneous backward heat conduction problem," Engineering Analysis with Boundary Elements, vol. 34, no. 9, pp. 785-792, 2010.

[13] T. Jiang, M. Li, and C. S. Chen, "The method of particular solutions for solving inverse problems of a nonhomogeneous convection-diffusion equation with variable coefficients," Numerical Heat Transfer A: Applications, vol. 61, no. 5, pp. 338352, 2012.

[14] T. S. Jiang, Z. L. Jiang, and K. Jospeh, "A numerical method for one-dimensional time-dependent Schrodinger equation using radial basis functions," International Journal of Computational Methods, vol. 10, no. 2, Article ID 1341010, 12 pages, 2013.

[15] G. R. Liu, Mesh Free Method: Moving Beyond the Finite Element Methods, CRC Press, Boca Raton, Fla, USA, 2002.

[16] G. R. Liu and M. B. Liu, Smooth Pariticle Hydrodynamics-A Meshfree Particle Method, World Scientific, Singapore, 2003. 
[17] S. N. Atluri and S. B. Shen, The Meshless Local PetrovGalerkin(MLPG) Method, Tech Science Press, Encino, Calif, USA, 2002.

[18] G. R. Liu and Y. T. Gu, An Introduction to Meshfree Methods and Their Programming, Springer, New York, NY, USA, 2005.

[19] M. Griebel and M. A. Schweitzer, Meshfree Methods for Partial Differential Equations III, Springer, New York, NY, USA, 2006.

[20] G. E. Fasshauer, Meshfree Approximation Methods with MAT$L A B$, Illinois Institute of Technology, Chicago, Ill, USA, 2008.

[21] A. J. Terrira, E. J. Kansa, G. E. Fasshauer, and V. M. Leitåo, Progress on Meshless Methods, New York, NY, USA, Springer, 2009.

[22] R. Schaback, "Error estimates and condition numbers for radial basis function interpolation," Advances in Computational Mathematics, vol. 3, no. 3, pp. 251-264, 1995.

[23] Z. M. Wu and R. Schaback, "Local error estimates for radial basis function interpolation of scattered data," IMA Journal of Numerical Analysis, vol. 13, no. 1, pp. 13-27, 1993. 


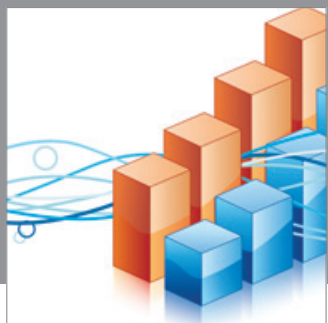

Advances in

Operations Research

mansans

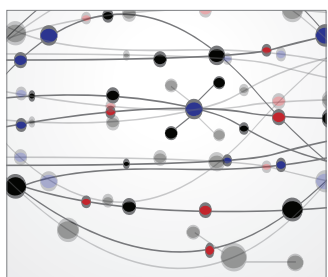

The Scientific World Journal
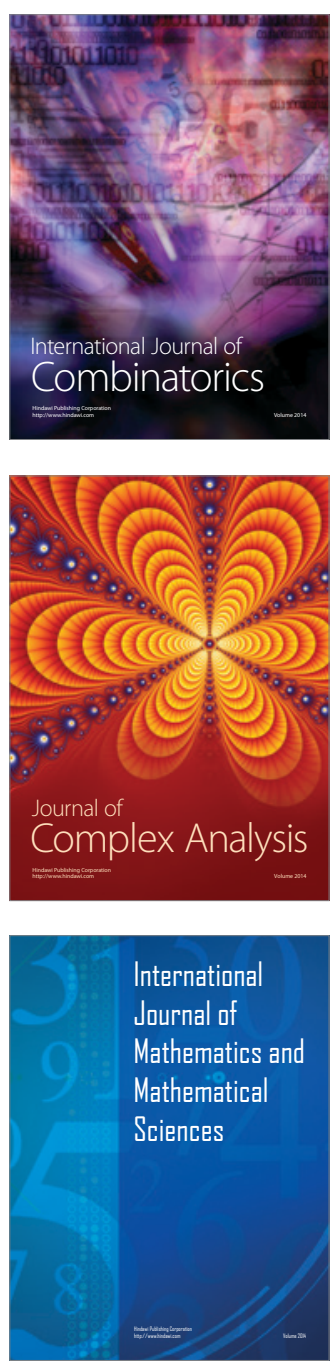
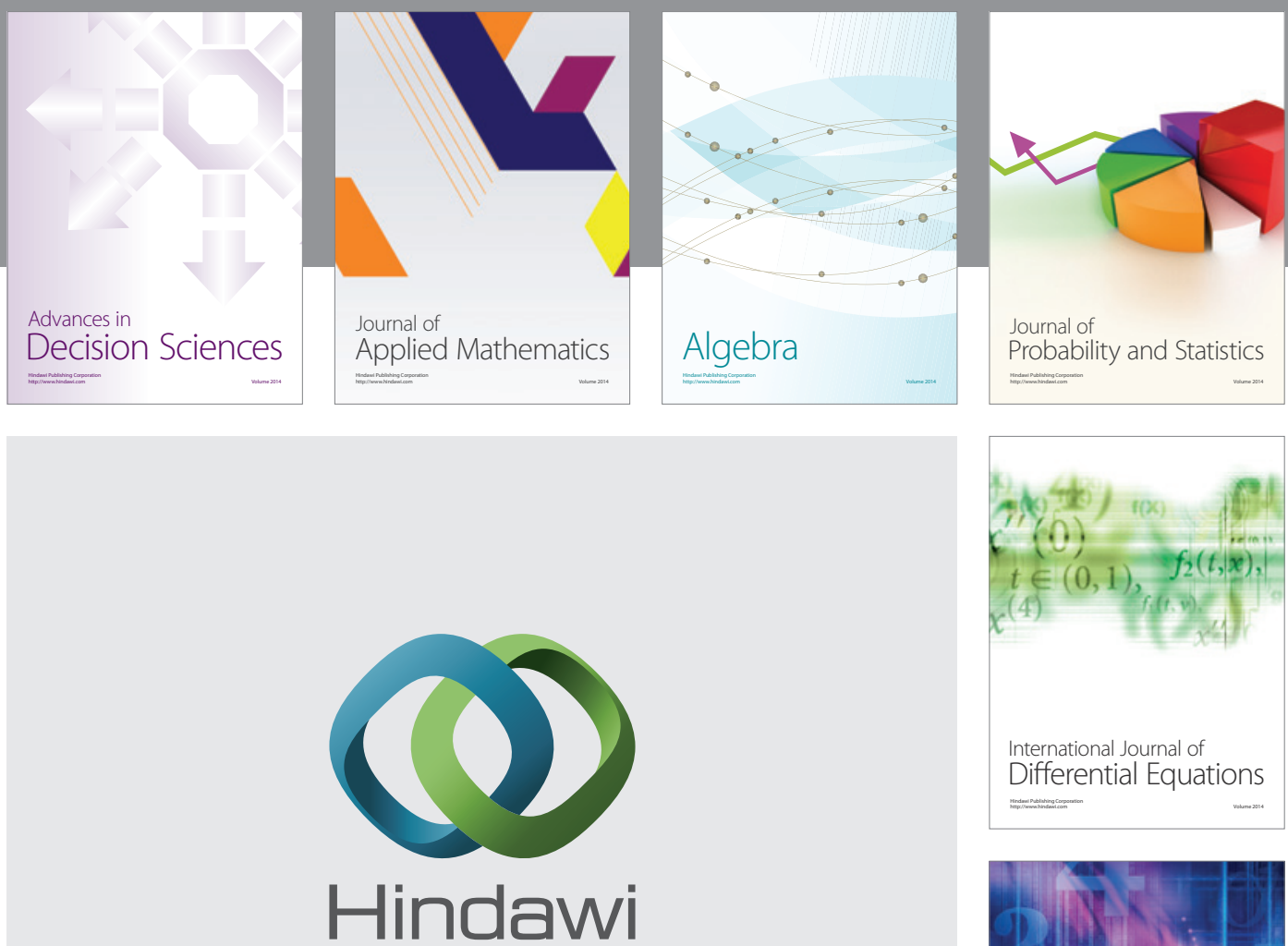

Submit your manuscripts at http://www.hindawi.com
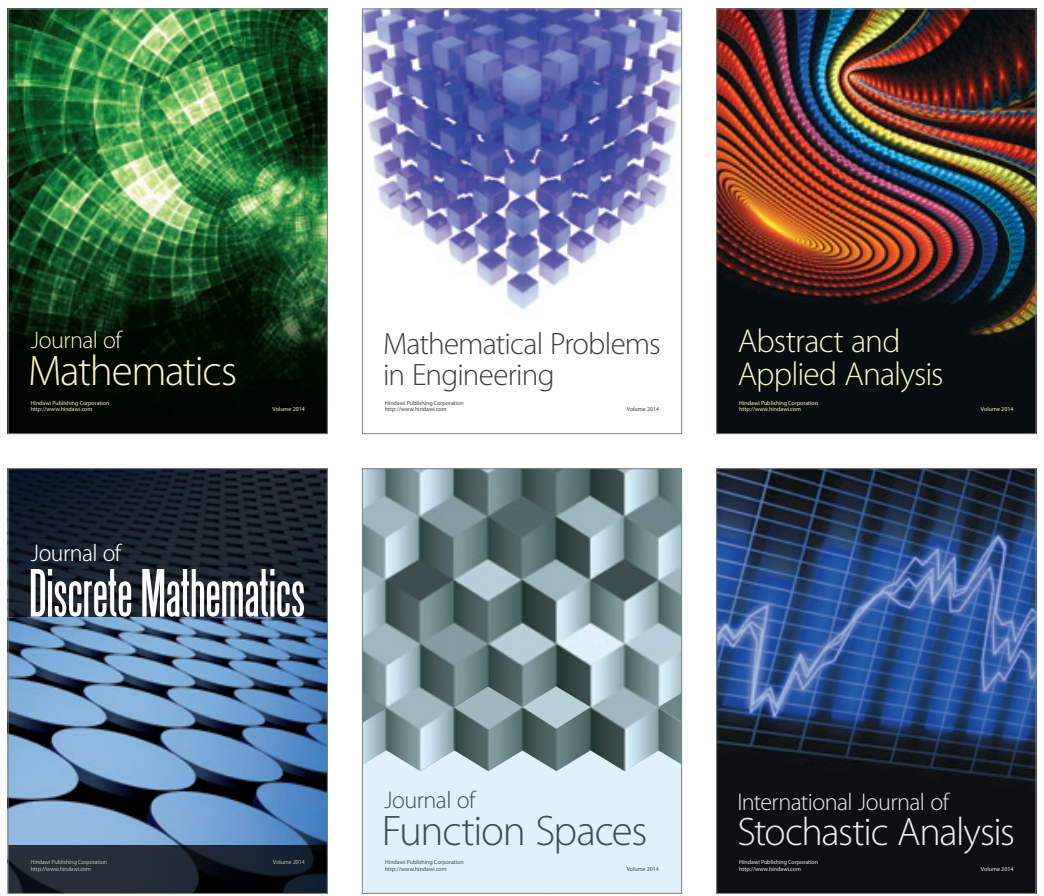

Journal of

Function Spaces

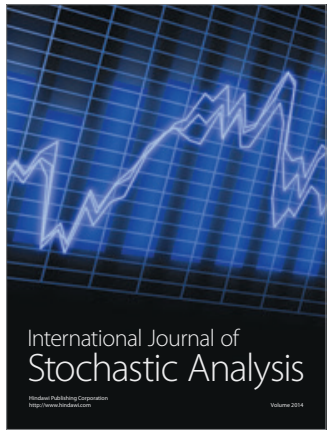

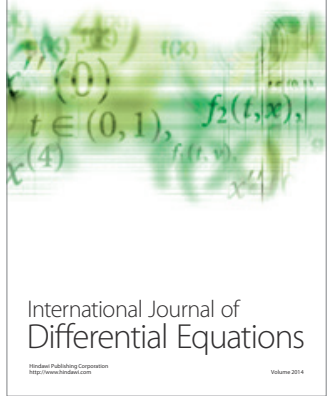
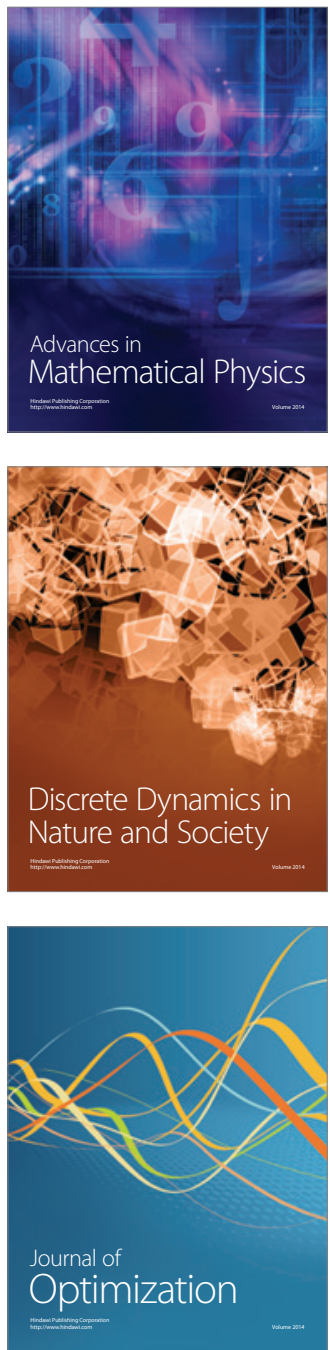